Pacific Journal of Mathematics

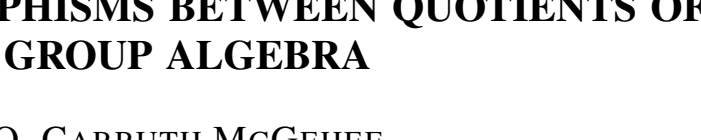




\section{CERTAIN ISOMORPHISMS BETWEEN QUOTIENTS OF A GROUP ALGEBRA}

\section{O. Carruth McGehee}

Let $T$ be the circle group, considered as the additive group of the real numbers modulo $2 \pi$. Let $A=A(T)$, the Banach algebra of functions on $T$ which have absolutely convergent Fourier series, with the norm of $f$ in $A$ equal to $\sum_{n}|\hat{f}(n)|$. If $E$ is a closed subset of $T$, we denote by $A(E)$ the quotient algebra $A / I(E)$, where $I(E)$ is the closed ideal consisting of those functions in $A$ which vanish on $E$. This paper presents a procedure for constructing perfect sets $E$ and $F$, which are not Helson sets, and a map $\varphi: F \rightarrow E$ inducing an isomorphism of $A(E)$ into $A(F)$. Thereby we shall obtain cases of an isomorphism of norm one, where $\varphi$ is the restriction to $F$ of a discontinuous character of $T$, composed with a rotation. In general, our $\varphi$ will be such a restriction at least on a dense subset of $F$, with the norm of the isomorphism not necessarily equal to one.

In the course of this construction we impose a condition of "arithmetic thinness" on the set $F$. As we shall prove, this condition is sufficient to imply that $F$ is a set of uniqueness.

Beurling and Helson [2] established that every automorphism of the algebra $A$ arises from a rigid motion of the circle-the composition of a rotation, $x \rightarrow x+x_{0}$, and a reflection, $x \rightarrow x$ or $x \rightarrow-x$. One may consider the problem of characterizing the cases in which a homeomorphism $\varphi$ of one closed set $F$ onto another, $E$, induces an isomorphism of $A(E)$ into $A(F)$. The methods of [2] may be modified to show that if $E$ and $F$ are intervals, then $\varphi(x)=r x+x_{0}$, where $r$ and $x_{0}$ are real; but these methods do not solve the problem for more general sets. DeLeeuw and Katznelson [4] showed that whenever the norm of an isomorphism of $A(E)$ into $A(F)$ is equal to one, it must arise from a map $\varphi: F \rightarrow E$ which is the restriction to $F$ of a character (an additive function of $T$ into $T$ ) composed with a rotation; and that if $F$ is "thick" in one of several senses, then this character must be a continuous one: $\varphi(x)=n x+x_{0}$, where $x_{0}$ is real and $n$ is an integer.

Let us call the map $\varphi: F \rightarrow E$ trivial if, near each point of $F$, it is equal to the restriction of a function $r x+x_{0}$, where $r$ and $x_{0}$ are real. What we shall show, in this terminology, is that there exist cases of a nontrivial $\varphi$ inducing an isomorphism of $A(E)$ into $A(F)$, where $E$ and $F$ are not Helson sets. Still, no such case is known in which $F$ is a set of multiplicity. 
2. Notation and definitions. The dual group of $T$ is $T^{\wedge}=Z$, the group of integers; and $A$ is the Gel'fand representation of $L^{1}(Z)$ (cf. [11], Ch. 1 and [7], App. I-IV). For $f \in A$, we let

$$
\hat{f}(n)=\frac{1}{2 \pi} \int_{0}^{2 \pi} f(x) e^{-i n x} d x,
$$

so that $f(x)=\sum_{n} \hat{f}(n) e^{i n x}$ and the $A$-norm is $\|f\|_{A}=\sum_{n}|\hat{f}(n)|$. The dual of the Banach space $A=L^{1}(Z)^{\wedge}$ is $P M=L^{\infty}(Z)^{\wedge}$; each functional $S \in P M$ is called a pseudomeasure. Letting $(f(x), S)$ or $(f, S)$ denote the value of $S$ at $f$, we set

$$
\hat{S}(n)=\overline{\left(e^{i n x}, S\right)} ; \quad(f, S)=\sum_{n} \hat{f}(n) \overline{\hat{S}(n)} .
$$

The pseudomeasure norm is $\|S\|_{P_{M}}=\sup _{n}|\hat{S}(n)|$.

Let $C=C(T)$, the Banach space of the continuous functions on the circle, with the usual norm; $\|f\|_{0} \leqq\|f\|_{A}$ if $f \in A$. The dual space of $C$ is $M=M(T)$, the space of the finite, regular, complex-valued measures $\mu$, with the value of $\mu$ at $f$ given by

$$
(f, \mu)=\frac{1}{2 \pi} \int_{0}^{2 \pi} f(x) \overline{d \mu(x)}
$$

and norm $\|\mu\|_{\mu}$ equal to the total mass. The Fourier-Stieltjes transform of $\mu \in M$ is the function on $Z$

$$
\hat{\mu}(n)=\frac{1}{2 \pi} \int_{0}^{2 \pi} e^{-i n x} d \mu(x) .
$$

Now $\mu \in P M$, with $\|\mu\|_{P_{M}} \leqq\|\mu\|_{M}$ and

$$
(f, \mu)=\sum_{n} \hat{f}(n) \overline{\hat{\mu}(n)} \text { for } f \in A .
$$

The inclusions $A \subset C$ and $M \subset P M$ are proper.

Two closed subspaces of $P M$ are of special interest. One is the space of pseudofunctions

$$
P F=C_{0}(Z)^{\wedge}=\left\{S \in P M: \lim _{|n| \rightarrow \infty} \hat{S}(n)=0\right\} ;
$$

note that the dual of $P F$ is $A$. The other is $A P=A P(Z)^{\wedge}$, consisting of the pseudomeasures $S$ whose transforms $\hat{S}$ are almost periodic functions on the integers. $A P(Z)$ is the closed space generated by the characters $\left\{e^{i n x}: x \in T\right\}$ of $Z$. For each $x \in T, e^{i n x}$ is a character on $Z$ and is the Fourier-Stieltjes transform of the measure $\delta_{x}$ which places mass 1 at $x$. Thus $A P$ contains all the measures with countable support in $T$. 
Sets of uniqueness and sets of multiplicity. (Cf. [7], App. I-IV and Ch. V; and [13], Ch. IX.) For an arbitrary $S \in P M$, consider the two series

$$
S_{1}(z)=\sum_{n=0}^{\infty} \hat{S}(n) z^{n}, S_{2}=-\sum_{n=-\infty}^{-1} \hat{S}(n) z^{n}
$$

The first represents a holomorphic function for $D_{1}=\{|z|<1\}$, the second for $D_{2}=\{|z|>1\}$. A point $x \in T$ is called a regular point of $S$ if $e^{i x}$ has a plane neighborhood $U$ on which there is a holomorphic function agreeing with $S_{1}$ on $D_{1} \cap U$ and with $S_{2}$ on $D_{2} \cap U$. The set of regular points of $S$, an open set, is called the null set of $S$. Its complement is the support of $S$; any set containing the support of $S$ is said to support, or carry $S$.

For a closed set $E \subset T$, let

$$
\begin{aligned}
& P M(E)=\{S \in P M: E \text { carries } S\}, \\
& M(E)=M \cap P M(E), \\
& P F(E)=P F \cap P M(E) .
\end{aligned}
$$

If $P F(E) \neq\{0\}, E$ is called a set of multiplicity; otherwise, a set of uniqueness. If $P F \cap M(E) \neq\{0\}, E$ is a set of multiplicity in the strict sense; otherwise a set of uniqueness in the broad sense. A set of uniqueness in the broad sense may be also a set of multiplicity; for a proof see [10], sections 1 and 3, or [9].

For $S \in P F$, a point $x \in T$ is a regular point if and only if the series $\sum_{n=-\infty}^{\infty} \hat{S}(n) e^{i n x}$ converges to zero throughout a neighborhood of $x$. Thus a closed set $E$ is a set of uniqueness if and only if there exists no nonzero pseudofunction $S$ such that $\sum_{n=-\infty}^{\infty} \widehat{S}(n) e^{i n x}$ converges to zero everywhere in the complement of $E$.

Quotient algebras. (Cf. [7], Ch. IX, X, XI.) Let $E$ be a closed subset of $T$ and let $I(E)$ be the closed ideal in $A$ consisting of the functions which vanish on $E$. Let $A(E)$ denote the quotient algebra $A / I(E)$, with the usual quotient norm:

$$
\|f\|_{\Delta(E)}=\inf \left\{\|f+g\|_{\Delta}: g \in I(E)\right\} .
$$

We may consider $A(E)$ as the algebra of restrictions to $E$ of functions in $A$, the restriction algebra of $E$.

The Banach space dual of $A(E)$ is

$$
N(E)=\{S \in P M:(f, S)=0 \quad \text { if } \quad f \in I(E)\} .
$$

The norm of $S \in N(E)=A(E)^{*}$ is precisely the pseudomeasure norm of $S$ : 


$$
\|S\|_{N(E)}=\|S\|_{P_{M}} \text { for } S \in N(E) \text {. }
$$

Similarly, let $C(E)$ be the algebra of restrictions to $E$ of functions in $C$;

$$
\|f\|_{\sigma_{(E)}}=\max \{|f(x)|: x \in E\} \quad \text { for } \quad f \in C(E) .
$$

The Banach space dual of $C(E)$ is $M(E)$;

$$
\|\mu\|_{M(E)}=\|\mu\|_{M} \text { for } \mu \in M(E) .
$$

In general,

$$
\begin{aligned}
& A(E) \subset C(E) ;\|f\|_{\sigma_{(E)}} \leqq\|f\|_{\boldsymbol{A}(E)} \text { if } \quad f \in A(E) ; \\
& M(E) \subset N(E) ;\|\mu\|_{P_{M}} \leqq\|\mu\|_{\boldsymbol{M}} \text { if } \quad \mu \in M(E) .
\end{aligned}
$$

The set $E$ is called a Helson set if $A(E)=C(E)$, that is, if every continuous function on $E$ is the restriction to $E$ of a function in $A$. A set $E$ is a Helson set if and only if there is a constant $c>0$ such that

$$
\|\mu\|_{M} \leqq c\|\mu\|_{P_{M}} \text { for } \quad \mu \in M(E) .
$$

The set $E$ is a set of synthesis if $I(E)$ is the only closed ideal whose hull is $E$; or, equivalently, if $N(E)=P M(E)$. This equality does not always hold.

3. A sketch of the procedure. Let $\Phi$ denote an isomorphic mapping of $A(E)$ into $A(F)$. We then have

$$
\|\Phi f\|_{\boldsymbol{A}(F)} \leqq\|\Phi\|\|f\|_{\boldsymbol{A}(E)} \text { for } f \in A(E) .
$$

If, as we assume, the functions in the image of $A(E)$ separate points in $F$ and do not all vanish at any point of $F$, then the mapping $\Phi$ must arise from a homeomorphism $\varphi: F \rightarrow E$ by the rule

$$
\Phi f(x)=f(\varphi(x)) \text { for } \quad x \in F
$$

(cf. [8], p. 76). It is evident from (2.1) and (2.2) that for every integer $n$, the function $e^{i n x}$ on $E$ has $A(E)$-norm 1. Therefore its image $e^{i n \varphi(x)}$ in $A(F)$ has $A(F)$-norm no greater than $\|\Phi\|$. Conversely, for every homeomorphism $\varnothing$ of $F$ onto $E$ which is in $A(F)$, such that $\left\|e^{i n \varphi(x)}\right\|_{\boldsymbol{A}(\boldsymbol{F})}$ is bounded uniformly in $n$, the rule (3.1) defines an isomorphism

$$
\Phi: A(F) \rightarrow A(E)
$$

with norm $\|\Phi\|=\sup _{n}\left\|e^{i n \varphi(x)}\right\|_{\boldsymbol{A}(F)}$.

The adjoint map of $\Phi$,

$$
\Phi^{*}: N(F) \rightarrow N(E),
$$


is defined by the condition:

$$
\left(f, \Phi^{*} S\right)=(\Phi f, S) \text { for } f \in A(E) .
$$

Our plan is as follows. We shall describe two sets $E$ and $F$ in $[0,2 \pi)$ and a bicontinuous map $\varphi$ taking $F$ onto $E$. The set $F$ will be the intersection $\bigcap_{k=1}^{\infty} F^{k}$, where $F^{k}$ is the union of $J(k)$ closed intervals; $F_{k}$ will denote the set of left-hand endpoints of these intervals: $F_{k}=\left\{s_{1}, \cdots, s_{J(k)}\right\}$. For $E$, the sets $E^{k}$ and $E_{k}=\left\{r_{1}, \cdots, r_{J(k)}\right\}$ will be defined similarly. For each $k$, the map $\varphi$ will take $F_{k}$ onto $E_{k}: \varphi\left(s_{j}\right)=r_{j}$ for $j=1, \cdots, J(k)$. We shall require that $\varphi$ preserve arithmetic relations on $F_{k}$; that is, whenever $u_{1}, \cdots, u_{J(k)}$ are integers such that $\sum_{j=1}^{J(k)} u_{j} s_{j}=0$ modulo $2 \pi$, then also $\sum_{j=1}^{J(k)} u_{j} r_{j}=0$ modulo $2 \pi$.

We shall place on $F$ an "arithmetic thinness" condition, requiring in particular that it be so "close" to its finite subsets $F_{k}$ that every $S \in P M(F)$ is the limit-in the $A$, or weak*, topology of $P M$-of a sequence $\left\{\mu_{k}\right\}$ of measures supported by the finite sets $F_{k}$. The condition will imply that $F$ is a set of uniqueness.

We shall also place on $E$ a relatively mild thinness condition.

Since $\varphi$ is continuous, the map $\Phi$ takes $C(E)$ onto $C(F)$, and its adjoint $\Phi^{*}$ takes $M(F)$ onto $M(E)$-both isometrically. But as we shall show, the conditions placed on $\varphi, F$, and $E$ imply that $\Phi^{*}$ extends to a continuous map of $N(F)$ into $N(E)$, that $\phi \in A(F)$, and that the norms $\left\|e^{i n \varphi(x)}\right\|_{\boldsymbol{A}^{(F)}}$ are bounded uniformly in $n$. Consequently $\Phi$ maps $A(E)$ isomorphically into $A(F)$.

4. Lemmas about finitely supported measures. In the present section we consider the case of a finite set $F_{0}=\left\{s_{j}: j=1, \cdots, J\right\}$ of $J$ distinct points, and the measures $\mu \in M\left(F_{0}\right)$. Let $\mu$ assign mass $a_{j}$ to the point $s_{j}$. The Fourier-Stieltjes transform of $\mu$ is

$$
\widehat{\mu}(n)=\sum_{j=1}^{J} a_{j} \exp \left(-i n s_{j}\right) .
$$

Its supremum is the pseudomeasure norm $\|\mu\|_{P_{M}}$ of $\mu$.

Every function on a finite set $F_{0}$ is the restriction to $F_{0}$ of a function in $A$, which is to say, a finite set is a Helson set; the $C\left(F_{0}\right)$ and $A\left(F_{0}\right)$ norms are equivalent, as of course are the $M\left(F_{0}\right)$ and $N\left(F_{0}\right)$ norms. The constant of this equivalence depends on the set. For an arithmetic sequence $\{a+j b: j=1, \cdots, J\}(b \neq 0)$, the constant is of the order of $J^{1 / 2}$ (cf. [7], Lemma 2, p. 134, or [13], V. 4.7). As we are about to show, it is never greater than $J^{1 / 2}$.

Definition. Let $B\left(s_{1}, \cdots, s_{J}\right)$ be the smallest constant $B$ such that 


$$
\sum_{j=1}^{J}\left|a_{n}\right|=\|\mu\|_{M} \leqq B\|\mu\|_{P_{M}} \text { for every } \mu \in M\left(F_{0}\right)
$$

Lemma 1 . In every case, $B\left(s_{1}, \cdots, s_{J}\right) \leqq J^{1 / 2}$.

Proof.

$$
\begin{aligned}
|\hat{\mu}(n)|^{2} & =\sum_{j=1}^{J} \sum_{i=1}^{J} a_{i} \bar{a}_{j} \exp \left[i n\left(s_{j}-s_{i}\right)\right] \\
& =\sum_{j=1}^{J}\left|a_{j}\right|^{2}+\sum_{i \neq j} a_{i} \bar{a}_{j} \exp \left[i n\left(s_{j}-s_{i}\right)\right] ; \\
\|\mu\|_{P M}^{2} & =\sup _{n}|\hat{\mu}(n)|^{2} \geqq \lim _{N \rightarrow \infty}(2 N+1)^{-1} \sum_{n=-N}^{N}|\hat{\mu}(n)|^{2} \\
& =\sum_{j=1}^{J}\left|a_{j}\right|^{2} \geqq J^{-1}\left(\sum_{j=1}^{J}\left|a_{j}\right|\right)^{2},
\end{aligned}
$$

the last line by the Cauchy inequality. The lemma is proved.

In general, $B\left(s_{1}, \cdots, s_{J}\right)$ depends upon the nature of the arithmetic relations among the $s_{j}$ 's; a relation is an equation

$$
\left\|\sum_{j=1}^{J} u_{j} s_{j}\right\|=0
$$

where the $u_{j}$ 's are integers and $\|x\|$ denotes the distance from the real number $x$ to the nearest integral multiple of $2 \pi$. If there are no relations among the $s_{j}$ 's, that is, if they are independent modulo $2 \pi$ over the rationals, then $B\left(s_{1}, \cdots, s_{\mathrm{J}}\right)=1$, by Kronecker's Theorem (cf. [7], App. V).

The transform (4.1) is an almost periodic function on the integers: for every $\varepsilon>0$, the integers $p$ such that

$$
|\widehat{\mu}(m+p)-\widehat{\mu}(m)| \leqq \varepsilon\|\mu\|_{P_{M}} \text { for every } m
$$

are relatively dense; that is, there is an $N$ such that every set of $2 N$ consecutive integers contains such a $p$. In particular,

$$
\max _{|n-m| \leqq N}|\widehat{\mu}(n)| \geqq(1-\varepsilon)\|\mu\|_{P M} \text { for every } m .
$$

The definition of almost periodicity is customarily stated with just " $\varepsilon$ " on the right-hand side of (4.2). Our version has the feature that the $N$ depends on $\varepsilon$ and the set $F_{0}$ but not on $\mu$. For let $m$ and $p$ be integers;

$$
\begin{aligned}
\mid \hat{\mu}(m+p) & -\hat{\mu}(m)|=| \sum_{j=1}^{J} a_{j}\left[\exp \left(-i(m+p) s_{j}\right)-\exp \left(-i m s_{j}\right)\right] \mid \\
& \leqq\left(\sum_{j=1}^{J}\left|a_{j}\right|\right) \max _{1 \leqq j \leqq J}\left|1-\exp \left(i p s_{j}\right)\right| \\
& \leqq B\left(s_{1}, \cdots, s_{J}\right)\|\mu\|_{P M} \cdot \max _{1 \leqq j \leqq J}\left\|p s_{j}\right\| .
\end{aligned}
$$


The solutions $p$ to the system of inequalities

$$
\left\|p s_{j}\right\|<\varepsilon / B, \quad j=1, \cdots, J
$$

are relatively dense, and the system does not involve $\mu$, so that $N$ may be selected as claimed. In particular, we have proved:

Lemma 2. Given $\varepsilon>0$, there is a number $N=N\left(s_{1}, \cdots, s_{J} ; \varepsilon\right)$ such that for every $\mu \in M\left(F_{0}\right)$,

$$
\max _{|n-m| \leqq N}|\widehat{\mu}(n)| \geqq(1-\varepsilon)\|\mu\|_{P_{M}} \text { for every } m \text {. }
$$

Note. There is no bound for $N$ depending on $J$ and $\varepsilon$ alone; the set of points $\left\{s_{1}, \cdots, s_{J}\right\}$ is critical.

Any two finite sets with the same number of points have isomorphic restriction algebras. Let

$$
\begin{gathered}
F_{0}=\left\{s_{j}: j=1, \cdots, J\right\}, \quad E_{2}=\left\{r_{j}: j=1, \cdots, J\right\}, \\
\varphi\left(s_{j}\right)=r_{j} .
\end{gathered}
$$

Then $\varphi$ maps $F_{0}$ onto $E_{0}$ and induces an isomorphism $\Phi$ of $A\left(E_{0}\right)$ onto $A\left(F_{0}\right)$ as in (3.1). For $\mu \in M\left(F_{0}\right)$ let $\mu^{\sharp}$ denote $\Phi^{*} \mu$, which is the measure on $E_{0}$ such that

$$
\mu^{\sharp}\left(r_{j}\right)=\mu\left(s_{j}\right) .
$$

The norm of $\Phi^{*}$ is the supremum of the ratio $\left\|\mu^{\sharp}\right\|_{P_{M}} /\|\mu\|_{P_{M}}$ for $\mu \in M\left(F_{0}\right)$. We know that this ratio is bounded by $J^{1 / 2}$, because

$$
\left\|\mu^{\#}\right\|_{P M} \leqq\left\|\mu^{\#}\right\|_{M},\left\|\mu^{*}\right\|_{M}=\|\mu\|_{M}
$$

by the definition of $\mu^{\sharp}$, and $\|\mu\|_{M} \leqq J^{1 / 2}\|\mu\|_{P_{M}}$ by Lemma 1 .

Lemma 3. If $\varphi$ preserves arithmetic relations on the set $\left\{s_{1}, \cdots, s_{J}\right\}$, so that

$$
\left\|\sum_{j=1}^{J} u_{j} s_{j}\right\|=0 \Rightarrow\left\|\sum_{j=1}^{J} u_{j} r_{j}\right\|=0
$$

for all integral $\left(u_{1}, \cdots, u_{J}\right)$, then the range of $\hat{\mu}$ is dense in that of $\hat{\mu}^{\xi}$. In particular,

$$
\left\|\mu^{\#}\right\|_{P_{M}} \leqq\|\mu\|_{P M} \quad \text { for } \quad \mu \in M\left(F_{0}\right) .
$$

Proof. By Kronecker's Theorem (cf. [3], p. 53 or p. 99) we know that the condition (4.3) insures that for every $\varepsilon$ and $m$, the inequalities

$$
\left\|n s_{j}-m r_{j}\right\|<\varepsilon, \quad j=1, \cdots, J
$$


can always be solved simultaneously for $n$. Since

$$
\begin{aligned}
\mid \hat{\mu}(n) & -\hat{\mu}^{\ddagger}(m) \mid=\left\|\sum_{j=1}^{J} \mu\left(s_{j}\right)\left[\exp \left(-i n s_{j}\right)-\exp \left(i m r_{j}\right)\right]\right\| \\
& \leqq\|\mu\|_{M} \cdot \max _{1 \leqq j \leqq J}\left\|n s_{j}-m r_{j}\right\|,
\end{aligned}
$$

the lemma follows.

REMARK. We should prefer a weaker, but still convenient, hypothesis in Lemma 3, giving the weaker conclusion that for some $c \geqq 1$,

$$
\left\|\mu^{\sharp}\right\|_{P M} \leqq c\|\mu\|_{P M} \text { for } \quad \mu \in M\left(F_{0}\right)
$$

- where both the hypothesis and the constant $c$ are independent of $J$. For example, perhaps it is true that if $(4.3)$ is required to hold only for those integral $\left(u_{1}, \cdots, u_{J}\right)$ with $\left|u_{j}\right| \leqq 2$ (or some other bound), then (4.4) follows for some $c$. We also should like to have estimates of the function $N$, in Lemma 2, better than those provided by the methods of Diophantine approximation theory. But we leave these questions unanswered.

5. Construction of $E, F$, and $\varphi$. We shall now give in detail our conventions for describing the sets $E$ and $F$ and the map $\varphi: F \rightarrow E$ which were discussed in $\S 3$. We shall describe closed perfect subsets $E$ and $F$ of the interval $[0,2 \pi)$, and a homeomorphism $\varphi$ mapping $F$ onto $E$.

Let $F=\bigcap_{k=1}^{\infty} F^{k}$, where $F^{k}$ is the union of $J(k)$ pairwise disjoint closed intervals, each with length $d_{k}>0$. We assume once and for all that

$$
\lim _{k \rightarrow \infty} J(k)=\infty, \quad \lim _{k \rightarrow \infty} J(k) d_{k}=0 .
$$

Let $F_{k}$ denote the set of the left-hand endpoints $s_{j}$ of the intervals making up $F^{k}$ :

$$
F_{k}=\left\{s_{1}, \cdots, s_{\mathbf{J}(k)}\right\} .
$$

Thus $F^{k}$ is determined by the selection of the set $F_{k}$ and the number $d_{k}$. In making this selection, we require that

$$
s_{1}<s_{2}<\cdots<s_{J(1)}
$$

and that for $k \geqq 2$, 
and

$$
\begin{gathered}
F_{k-1} \subset F_{k}=\left\{s_{1}, \cdots, s_{J(k-1)}, \cdots, s_{\boldsymbol{J}(k)}\right\} ; \\
s_{\boldsymbol{J}(k-1)+1}<\cdots<s_{\boldsymbol{J}(k)} ;
\end{gathered}
$$

$$
F^{k} \subset F^{k-1} \text {. }
$$

Thus every point $s_{j}$ in $F_{k}$ but not in $F_{k-1}(J(k-1)<j \leqq J(k))$ lies in the interval $\left[s_{i}, s_{i}+d_{k-1}-d_{k}\right]$ for some $s_{i} \in F_{k-1}(1 \leqq i \leqq J(k-1))$.

We further require that for every $k$, the points of $F_{k}$ are at least $2 d_{k}$ apart, modulo $2 \pi$. Thus not only are the intervals of $F^{k}$ disjoint; but also, each of the intervals contiguous to $F^{k}$ in $[0,2 \pi]$ has length no less than $d_{k}$.

Now let $E$ be a set constructed in the same manner, except with different choices of the numbers $d_{k}$ and the sets of endpoints, and with different notation, as follows: $E=\bigcap_{k=1}^{\infty} E^{k}$, where $E^{k}$ is the union of $J(k)$ intervals with length $d_{k}^{\prime}$ and left-hand endpoints $r_{j} ; E_{k}=$ $\left\{r_{1}, \cdots, r_{J(k)}\right\}$. We will again have

$$
\lim _{k \rightarrow \infty} J(k) d_{k}^{\prime}=0 \text {. }
$$

We shall place the points of $E_{k}$ in correspondence with those of $F_{k}$, in the following sense: for $k \geqq 2$, we select the points $r_{j}$ for $J(k-1)<j \leqq J(k)$ in such a way that, for each $i=1, \cdots, J(k-1)$, the number of these $r_{j}^{\prime}$ 's placed in the interval $\left[r_{i}, r_{i}+d_{k-1}^{\prime}-d_{k}^{\prime}\right]$ equals the number of $s_{j}$ 's (with $J(k-1)<j \leqq J(k)$ ) appearing in the interval $\left[s_{i}, s_{i}+d_{k-1}-d_{k}\right]$.

For each $k$, let $\varphi_{k}$ be the continuous increasing function which maps $[0,2 \pi]$ onto itself such that

$$
\varphi_{k}(0)=0, \quad \varphi_{k}\left(s_{j}\right)=r_{j}(1 \leqq j \leqq J(k)), \quad \varphi_{k}(2 \pi)=2 \pi ;
$$

and which is linear on each interval contiguous to the set $\left\{0, s_{1}, \cdots\right.$, $\left.s_{J(k)}, 2 \pi\right\}$. By (5.1) and (5.2), the sequences $\left\{\varphi_{k}\right\}$ and $\left\{\varphi_{k}^{-1}\right\}$ converge uniformly as $k \rightarrow \infty$ to functions $\varphi$ and $\varphi^{-1}$ respectively; which then must be continuous, each the inverse of the other. Therefore $\varphi$ maps $F$ homeomorphically onto $E$.

6. Approximating pseudomeasures by finitely supported measures.

Lemma 4. Let $F$ be the set constructed in $\S 5$. By a method to be explained below, it is possible to associate with each $S \in P M(F)$ a sequence of measures $\mu_{k} \in M\left(F_{k}\right)$, such that

$$
\left|\widehat{S}(n)-\hat{\mu}_{k}(n)\right| \leqq|n|\left(J(k) d_{k}\right)^{1 / 2}\|S\|_{P M} \text { for all } k, n \text {. }
$$

In particular, by (5.1), 


$$
\lim _{k \rightarrow \infty} \hat{\mu}_{k}(n)=\widehat{S}(n) \text { for all } n
$$

Proof. We shall follow Kahane and Salem ([7], p. 126). For each $k, F^{k}$ is the union of $J(k)$ closed intervals. Let us give them names and enumerate from left to right:

$$
I_{1}, I_{2}, \cdots, I_{J(k)} \text {. }
$$

Without loss of generality we may assume 0 to be the left-hand endpoint of $I_{1}$. Then the interval $[0,2 \pi]$ is the union of the sets

$$
I_{1}, I_{1}^{\prime}, I_{2}, I_{2}^{\prime}, \cdots, I_{J(k)}, I_{J(k)}^{\prime}
$$

where $I_{1}^{\prime}, \cdots, I_{J(k)}^{\prime}$ are the intervals contiguous to $F^{k}$ in $[0,2 \pi]$, listed from left to right.

Let $S \in P M(F)$ with $\hat{S}(0)=0$. The formal integral of $S$ is the $L^{2}$ function

$$
\sigma(x) \sim \sum_{n \neq 0} \frac{\widehat{S}(n)}{i n} e^{i n x}
$$

with norm

$$
\|\sigma\|_{2} \leqq\left(\sum_{n \neq 0} n^{-2}\right)^{1 / 2}\|S\|_{P_{M}}
$$

The function $\sigma(x)$ will be constant on each interval $I_{j}^{\prime}$. Let $\sigma_{k}(x)$ be the step function which on $I_{j} \cup I_{j}^{\prime}$ has the same constant value that $\sigma(x)$ has on $I_{j}^{\prime}$. In $\S 5$ we stipulated that each $I_{j}^{\prime}$ must have length no less than the length of $I_{j}$, which is $d_{k}$. Therefore

$$
\int_{F^{k}}\left|\sigma_{k}(x)\right|=\int_{F^{k}}\left|\sigma\left(x+d_{k}\right)\right|,
$$

and hence both the quantities $\int_{F^{k}}\left|\sigma_{k}(x)\right|$ and $\int_{F^{k}}|\sigma(x)|$ are majorized by $\left(J(k) d_{k}\right)^{1 / 2}\|\sigma\|_{2}$. The measure $\mu_{k}=d \sigma_{k}$ is supported by the finite set $F_{k}$, and

$$
\hat{S}(n)-\hat{\mu}_{k}(n)=\frac{i n}{2 \pi} \int_{0}^{2 \pi}\left[\sigma(x)-\sigma_{k}(x)\right] e^{-i n x} d x .
$$

Since the integrand is zero on the complement of $F^{k}$, we have

$$
\begin{aligned}
\mid \hat{S}(n) & -\hat{\mu}_{k}(n) \mid \leqq \frac{|n|}{2 \pi}\left(\int_{F^{k}}|\sigma(x)|+\int_{F^{k}}\left|\sigma_{k}(x)\right|\right) \\
& \leqq \frac{|n|}{\pi}\left(J(k) d_{k}\right)^{1 / 2}\|\sigma\|_{2},
\end{aligned}
$$

which with (6.3) implies (6.1). 
If $\hat{S}(0) \neq 0$, let $x$ be a point in $F_{1}$ (and hence in every $F_{k}$ ), and consider $T=S-\widehat{S}(0) \delta_{x}$ instead of $S$. Then $\hat{T}(n)=\hat{S}(n)-\widehat{S}(0) e^{-i n x}$, $\widehat{T}(0)=0$. Associate $\mu_{k}^{\prime}$ with $T$ by the above process; (6.1) will then hold for $S$ if we take $\mu_{k}=\mu_{k}^{\prime}+\widehat{S}(0) \delta_{x}$. The proof of Lemma 4 is complete.

7. A thinness condition for the set $F$. We shall now make use of Lemma 4 to study the implications of a certain thinness requirement, which we call

Condition I.

$$
\lim _{k \rightarrow \infty}\left(J(k) d_{k}\right)^{1 / 2} N\left(s_{1}, \cdots, s_{J(k)} ; \alpha\right)=0,
$$

where $0<\alpha<1$, and where $N$ is the function of Lemma 2. Condition I may be enforced in the construction of the set $F$ without restricting the quantity of arithmetic relations among the points $\left\{s_{j}\right\}$, since at each step, $d_{k}$ may be chosen after $N_{k}$ is evaluated. Let us illustrate that Condition I does not imply that $F$ is a Helson set. Let $\left\{p_{k}\right\}$ be a positive sequence, $\sum_{k=1}^{\infty} p_{k}<1$, and consider the set consisting of the sums $\left\{\sum_{k=1}^{\infty} \varepsilon_{k} p_{k}: \varepsilon_{k}=0\right.$ or 1$\}$. Such a set is called a symmetric set. By replacing $\left\{p_{k}\right\}$ with a subsequence tending to zero fast enough, we obtain a set satisfying Condition I. But no symmetric set can be a Helson set (cf. [7], Ch. XI, Th. VIII).

THEOREM 1. Let $F$ be a set constructed as in $\S 5$, obeying Condition I. If $S \in P M(F)$ and $\left\{\mu_{k}\right\}$ is the sequence associated with $S$ as in Lemma 4, then

$$
\lim \sup _{k \rightarrow \infty}\left\|\mu_{k}\right\|_{P_{M}} \leqq(1-\alpha)^{-1}\|S\|_{P_{M}} .
$$

Also,

$$
\lim \sup _{|n| \rightarrow \infty}|\widehat{S}(n)| \geqq(1-\alpha)\|S\|_{P_{M}} \text { for every } S \in P M(F) \text {. }
$$

Proof. For convenience let us write

$$
\begin{gathered}
N_{k}=N\left(s_{1}, \cdots, s_{J(k)} ; \alpha\right) ; \\
\varepsilon_{k}=N_{k}\left(J(k) d_{k}\right)^{1 / 2} .
\end{gathered}
$$

Then by (6.1),

$$
\left|\hat{S}(n)-\hat{\mu}_{k}(n)\right| \leqq \varepsilon_{k}|n| N_{k}^{-1}\|S\|_{P_{M}} \text { for all } k, n ;
$$

and by Condition I, $\lim _{k \rightarrow \infty} \varepsilon_{k}=0$. By the definition of $N_{k}$, there is an $n_{0}$ such that $\left|n_{0}\right| \leqq N_{k}$ and 


$$
\begin{aligned}
\left\|\mu_{k}\right\|_{P M}(1-\alpha) & \leqq\left|\hat{\mu}_{k}\left(n_{0}\right)\right| \leqq\left|\hat{S}\left(n_{0}\right)\right|+\varepsilon_{k}\|S\|_{P M} \\
& \leqq\left(1+\varepsilon_{k}\right)\|S\|_{P M} ;
\end{aligned}
$$

(7.1) follows.

Let $\eta>0$ and pick $m_{0}$ such that $\left|\widehat{S}\left(m_{0}\right)\right| \geqq\|S\|_{P M}(1-\eta)$. Let $k$ be large enough so that $\left|m_{0}\right| \leqq N_{k}$. There is an $n_{k}$ (cf. Lemma 2) between, say, $7 N_{k}$ and $9 N_{k}$ such that $\left|\hat{\mu}_{k}\left(n_{k}\right)\right| \geqq(1-\alpha)\left\|\mu_{k}\right\|_{P M H}$. So:

$$
\left|\hat{S}\left(n_{k}\right)\right| \geqq\left|\hat{\mu}_{k}\left(n_{k}\right)\right|-9 \varepsilon_{k}|| S \|_{P M} ;
$$

but

$$
\begin{aligned}
\left|\hat{\mu}_{k}\left(n_{k}\right)\right| & \geqq(1-\alpha)\left\|\mu_{k}\right\|_{P M} \geqq(1-\alpha)\left|\hat{\mu}_{k}\left(m_{0}\right)\right| \\
& \geqq(1-\alpha)\left(\left|\hat{S}\left(m_{0}\right)\right|-\varepsilon_{k}\|S\|_{P M}\right) \\
& \geqq(1-\alpha)\|S\|_{P_{M}}\left(1-\eta-\varepsilon_{k}\right) .
\end{aligned}
$$

So

$$
\left|\widehat{S}\left(n_{k}\right)\right|>\|S\|_{P_{M}}\left[(1-\alpha)\left(1-\eta-\varepsilon_{k}\right)-9 \varepsilon_{k}\right] .
$$

Since $n_{k} \geqq 7 N_{k}$ we know $\lim _{k \rightarrow \infty} n_{k}=\infty$. Therefore

$$
\lim \sup _{|n| \rightarrow \infty}|\hat{S}(n)| \geqq\|S\|_{P M}(1-\alpha)(1-\eta),
$$

where $\eta$ is arbitrary; (7.2) follows, and the theorem is proved.

By Theorem 1, Condition I has several important consequences for the set $F$, which we now list as corollaries.

CoRollary 1. For each $S \in P M(F)$, the associated sequence $\left\{\mu_{k}\right\}$ converges to $S$ in the $A$ topology of $P M$.

Proof. This result is evident from (6.2) and (7.1).

COROLlaRY 2. The set $F$ is a set of synthesis.

Proof. We need to show that $P M(F)=N(F)$. Let $S \in P M(F)$. Each $\mu_{k}$ is in $N(F)$, that is, $\left(f, \mu_{k}\right)=0$ for every $f \in I(F)$. But $(f, S)=\lim _{k \rightarrow \infty}\left(f, \mu_{k}\right)$ for every $f \in A$, by Corollary 1 . Therefore $(f, S)=0$ for every $f \in I(F)$, so $S \in N(F)$.

COROLlaRY 3. The set $F$ is a set of uniqueness.

Proof. The result (7.2) easily implies that

$$
\lim \sup _{|n| \rightarrow \infty}|\hat{S}(n)|>0 \text { for every } S \in P M(F) \text {. }
$$

CoRollary 4. If the sequence $\left\{B\left(s_{1}, \cdots, s_{J(k)}\right): k=1,2, \cdots\right\}$, 
where $B$ is the function of Lemma 1 , is bounded, then $F$ is a Helson set.

Proof. In this case (7.1) implies that the sequence $\left\{\left\|\mu_{k}\right\|_{\mu}\right\}$ is bounded. This fact, together with (6.2) or Corollary 1 proves that $\left\{\mu_{k}\right\}$ converges in the $C$ topology of $M$. It must converge to some $\mu \in M$, and $\mu=S$; thus $P M(F)=M(F)$ and $F$ is a Helson set. This result is due to Kahane and Salem ([7], p. 126).

CoRollary 5. If Condition I holds for every $\alpha>0$, then

$$
\begin{gathered}
\lim _{k \rightarrow \infty}\left\|\mu_{k}\right\|_{P_{M}}=\|S\|_{P_{M}} \\
\lim \sup _{|n| \rightarrow \infty}|\hat{S}(n)|=\|S\|_{P_{M}} \quad \text { for every } \quad S \in P M(F) .
\end{gathered}
$$

Proof. Statements (7.1) and (7.2) hold for every $\alpha>0$.

Remark. Let $B$ be a Banach space, $B^{*}$ the dual space, $\Gamma$ a subspace of $B^{*}$; and for $f \in B$ define

$$
\|f\|_{1}=\sup \left\{\frac{|(f, g)|}{\|g\|_{B^{*}}}: g \in \Gamma, g \neq 0\right\} .
$$

If this norm is equivalent to the $B$ norm in $B$, then of course $\Gamma$ is $B$-dense in $B^{*}$, but as Dixmier [5] pointed out, the converse is false. An illustration of this fact is provided by the set $F$ constructed by Rudin ([12], or [7], p. 103), which is not a Helson set but which has $\|\mu\|_{M}=\|\mu\|_{P_{M}}$ for all those $\mu \in M(F)$ which have finite support. The space $\Gamma$ consisting of these measures is $A(F)$-dense in $N(F)$ (as it is for arbitrary $F$ ), but the $A(F)$ norm is not equivalent to the norm $\|f\|_{1}$, which in this case equals the $C(F)$ norm.

In the case of the set $F$ of Theorem 1, however, the finitely supported measures are $A(F)$-sequentially dense in $N(F)$ and

$$
\|f\|_{1} \geqq\|f\|_{A(F)}(1-\alpha) \text { for } f \in A(F) .
$$

Even these conditions do not reflect the full strength of the approximation of $S \in N(F)$ by the sequence $\left\{\mu_{k}\right\}$; for we have the further fact that $\hat{S}$ is well approximated by $\hat{\mu}_{k}$ throughout an almost-period of $\hat{\mu}_{k}$.

8. An isomorphism of $A(E)$ into $A(F)$. To establish the isomorphism, we shall place the following three requirements on the set $F$, the set $E$, and the mapping $\varphi$, respectively: 


$$
\lim _{k \rightarrow \infty}\left(J(k) d_{k}\right)^{1 / 2} N\left(s_{1}, \cdots, s_{J(k)} ; \alpha\right)=0,
$$

where $0<\alpha<1$, and where $N$ is the function of Lemma 2;

Condition II.

$$
\sum_{k=1}^{\infty} d_{k}^{\prime} B\left(r_{1}, \cdots, r_{J(k+1)}\right)<\infty,
$$

where $B$ is the function of Lemma 1 ; and

Condition III.

$$
\left\|\sum_{j=1}^{J(k)} u_{j} s_{j}\right\|=0 \Rightarrow\left\|\sum_{j=1}^{J(k)} u_{j} r_{j}\right\|=0
$$

for all integers $u_{1}, \cdots, u_{J(k)}$ and for every $k$.

Condition II is a relatively mild requirement. By Lemma 1, it holds if

$$
\sum_{k=1}^{\infty} d_{k}^{\prime} J(k+1)^{1 / 2}<\infty \text {. }
$$

It is satisfied, for example, by a symmetric set of constant ratio $\xi<1 / 2$ :

$$
\left\{(1-\xi) \xi^{-1} \sum_{k=1}^{\infty} \varepsilon_{k} \xi^{k}: \varepsilon_{k}=0 \text { or } 1 \text { for each } k\right\} .
$$

To describe this set we may take $J(k)=2^{k}, d_{k}^{\prime}=\xi^{k}$.

THEOREM 2. Let the sets $F$ and $E$ and the mapping $\varphi$, constructed as in $\S 5$, obey Conditions I, II, and III, respectively. Then by the rule (3.1), the mapping $\Phi$ induces the isomorphism $\Phi$ of $A(E)$ into $A(F)$, with the norm no greater than $(1-\alpha)^{-1}$. If Condition I holds for every $\alpha>0$, the isomorphism is norm-decreasing.

Proof. Using (3.1) for $f \in C(E)$, we see that the homeomorphism $\varphi$ of $F$ onto $E$ induces the isometric isomorphisms

$$
\begin{aligned}
& \Phi: C(E) \rightarrow C(F) ; \\
& \Phi^{*}: M(F) \rightarrow M(E) .
\end{aligned}
$$

By Lemma 3, Condition III implies that the restrictions of $\Phi^{*}$ to measures on the sets of endpoints,

$$
\Phi^{*}: M\left(F_{k}\right) \rightarrow M\left(E_{k}\right), \quad k=1,2, \cdots,
$$

are continuous with respect to the pseudomeasure norms; in fact 


$$
\left\|\mu^{\sharp}\right\|_{P_{M}} \leqq\|\mu\|_{P_{M}} \quad \text { for } \quad \mu \in M\left(F_{k}\right), \quad k=1,2, \cdots,
$$

where $\mu^{\sharp}$ denotes $\Phi^{*} \mu$. We shall now show that if $S \in N(F)$, and $\left\{\mu_{k}\right\}$ is the sequence associated with $S$ by Lemma 4, then Condition II implies that the sequence $\left\{\hat{\mu}_{k}^{*}(m): k=1,2, \cdots\right\}$ is a Cauchy sequence for every $m$; and we shall then define $S^{\sharp}=\omega^{*} S$ by the conditions $\widehat{S}^{\sharp}(n)=\lim _{k \rightarrow \infty} \hat{\mu}_{k}^{\sharp}(n)$. Let

$$
a_{j}=\mu_{k}^{*}\left(r_{j}\right)=\mu_{k}\left(s_{j}\right), \text { for } 1 \leqq j \leqq J(k) .
$$

Similarly, let

$$
b_{i}=\mu_{k+1}^{*}\left(r_{i}\right)=\mu_{k+1}\left(s_{i}\right), \text { for } 1 \leqq i \leqq J(k+1) .
$$

Then

$$
\begin{aligned}
& \hat{\mu}_{k}^{\#}(m)=\sum_{j=1}^{J(k)} a_{j} \exp \left(-i m r_{j}\right) ; \\
& \hat{\mu}_{k+1}^{\sharp}(m)=\sum_{i=1}^{J(k+1)} b_{i} \exp \left(-i m r_{i}\right) \\
& =\sum_{j=1}^{J(k)} \sum\left\{b_{i} \exp \left(-i m r_{i}\right): r_{i}-r_{j}<d_{k}^{\prime}\right\} \\
& =\sum_{j=1}^{J(k)} \sum\left\{b _ { i } \left[\exp \left(-i m r_{j}\right)+\exp \left(-i m r_{i}\right)\right.\right. \\
& \left.\left.-\exp \left(-i m r_{j}\right)\right]: r_{i}-r_{j}<d_{k}^{\prime}\right\} \\
& =\widehat{\mu}_{k}^{\sharp}(m)+\sum_{j=1}^{J(k)} \sum\left\{b _ { i } \left[\exp \left(-i m r_{i}\right)\right.\right. \\
& \left.\left.-\exp \left(-i m r_{j}\right)\right]: r_{i}-r_{j}<d_{k}^{\prime}\right\} \text {, }
\end{aligned}
$$

since

$$
a_{j}=\sum\left\{b_{i}: r_{i}-r_{j}<d_{k}^{\prime}\right\}
$$

by the definition of $\mu_{k}$ and $\mu_{k+1}$. Therefore

$$
\begin{array}{r}
\left|\hat{\mu}_{k+1}^{\sharp}(m)-\hat{\mu}_{k}^{\sharp}(m)\right| \leqq \min \left\{2,|m| d_{k}^{\prime}\right\} \\
=\mathcal{J} \sum_{i=1}^{J(k+1)}\left|b_{i}\right| \\
=\mathcal{O}\left(d_{k}^{\prime} B\left(r_{1}, \cdots, r_{J(k+1)}\right)\right) \text { as } k \rightarrow \infty
\end{array}
$$

because

$$
\sum_{i=1}^{J(k+1)}\left|b_{i}\right|=\left\|\mu_{k+1}^{*}\right\|_{M} \leqq B\left(r_{1}, \cdots, r_{J(k+1)}\right)\left\|\mu_{k+1}^{\#}\right\|_{P_{M}}
$$

and $\left\|\mu_{k}^{*}\right\|_{P_{M K}}=\mathscr{O}\left(\|S\|_{P_{M}}\right)$ by (8.2) and (7.1). Therefore, Condition II on the set $E$ implies that $\left\{\hat{\mu}_{k}^{\#}(m): k=1,2, \cdots\right\}$ is a Cauchy sequence for each $m$. Let $\hat{S}^{\sharp}(m)$ be its limit. Then 


$$
\begin{aligned}
& \left|\hat{S}^{\sharp}(m)\right|=\left|\lim _{k \rightarrow \infty} \hat{\mu}_{k}^{\sharp}(m)\right| \leqq(1-\alpha)^{-1}\|S\|_{P_{\mathcal{M}}} ; \\
& \text { thus }\left\|S^{\sharp}\right\|_{P_{M}} \leqq(1-\alpha)^{-1}\|S\|_{P_{M}} \text {. }
\end{aligned}
$$

Since $S^{\sharp}=\lim _{k \rightarrow \infty} \mu_{k}^{\#}$ in the $A$ topology of $P M$, we know $S^{\sharp} \in N(E)$. The map $S \rightarrow S^{\#}$ is an extension of (8.1) to a continuous map of $N(F)$ into $N(E)$, with norm no greater than $(1-\alpha)^{-1}$.

To show that $\varphi$ induces an isomorphism of $A(E)$ into $A(F)$, it suffices to show that $e^{i \varphi} \in A(F)$. For then

$$
\left(S, e^{i m \varphi}\right)=\lim _{k \rightarrow \infty}\left(\mu_{k}, e^{i m \varphi}\right)=\widehat{S}^{\sharp}(m)
$$

and hence

$$
\begin{aligned}
& \left|\left(S, e^{i m \varphi}\right)\right| \leqq(1-\alpha)^{-1}\|S\|_{P_{M Y}} \text { for all } S \in N(F), \\
& \text { so }\left\|e^{i m \varphi}\right\|_{A(F)} \leqq(1-\alpha)^{-1} \text { for all } m .
\end{aligned}
$$

We already know that $\varphi$ induces a continuous linear function $G$ on $N(F)$ :

$$
G(S)=\hat{S}^{\sharp}(1)=\lim _{k \rightarrow \infty}\left(\mu_{k}, e^{i m \varphi}\right) .
$$

Since $A(F)$ is total over $N(F), G \in A(F)$ if and only if $G$ is continuous in the $A(F)$ topology of $N(F)$ ([6], V. 3.11). But $G$ is $A(F)$-continuous if and only if it is continuous in the relative $A(F)$ topology of the ball $\left\{S:\|S\|_{P_{M}} \leqq a\right\}$ for every $a>0$ ([6], V. 5.6). Therefore it suffices to show that for arbitrary $a$ and $\varepsilon$, there exist $N$ and $\eta>0$ such that:

$$
\begin{gathered}
\|S\|_{P M} \leqq a \text { and }|\hat{S}(n)|<\eta \text { for }|n| \leqq N \\
\Longrightarrow|G(S)|<\varepsilon .
\end{gathered}
$$

If $\|S\|_{P_{M}} \leqq a$, then by (8.2) and the definition of $N_{k}$,

$$
\begin{aligned}
\left|\left(\mu_{k}, e^{i m \varphi}\right)\right| & =\left|\hat{\mu}_{k}^{*}(1)\right| \leqq\left\|\mu_{k}^{*}||_{P_{M}} \leqq\right\| \mu_{k} \|_{P_{M}} \\
& \leqq(1-\alpha)^{-1} \max _{|n| \leqq N_{k}}\left|\hat{\mu}_{k}(n)\right|,
\end{aligned}
$$

which by (7.3) is

$$
\leqq(1-\alpha)^{-1}\left[\max _{|n| \leqq N_{k}}|\hat{S}(n)|+\varepsilon_{k} a\right]
$$

so by (8.3),

$$
|G(S)| \leqq \varepsilon / 2+(1-\alpha)^{-1} \max _{|n| \leqq N_{k}}|\widehat{S}(n)|
$$

for $k$ large enough; and if $N=N_{k}$ and $\eta \leqq \varepsilon(1-\alpha) / 2$, then (8.4) follows. The theorem is proved. 
REMARK. For the extension of $\Phi^{*}$ to a continuous map on $N(F)$, it would suffice to have

$$
\left\|\mu^{\#}\right\|_{P_{M}} \leqq c\|\mu\|_{P_{M}} \quad \text { for } \quad \mu \in M\left(F_{k}\right), \quad k=1,2, \cdots,
$$

for some $c \geqq 1$. Condition III seems too strong, since it gives not only (8.5) with $c=1$, but much more, by Lemma 3. But we prefer to state the theorem using Condition III, because it gives an explicit sufficient condition on the selection of the points $\left\{r_{j}\right\}$ and $\left\{s_{j}\right\}$; and we do not know of any essentially weaker condition that will yield (8.5).

9. Examples. To obtain an isomorphism of $A(E)$ and $A(F)$, we apply Theorem 2 twice, requiring that the triple $E, F, \varphi^{-1}$, as well as $F, E, \varphi$, obey requirements analogous to Conditions I, II, and III, respectively. Then $\varphi^{-1}$ will induce $\Phi^{-1}$, whose norm will not exceed $\left(1-\alpha^{\prime}\right)^{-1}$, say. If Condition I holds on $F$ and $E$ for every positive $\alpha$ and $\alpha^{\prime}$, respectively, then $A(E)$ and $A(F)$ will be isometrically isomorphic.

Let us point out an example. For $i=1$ and 2 , let $G_{i}$ be the symmetric set $\left\{\sum_{k=1}^{\infty} \varepsilon_{k} \xi_{k}^{(i)}: \varepsilon_{k}=0\right.$ or 1$\}$, where $\left\{\xi_{k}^{(i)}\right\}$ is a sequence of numbers independent over the rationals. If $\xi_{k}^{(i)} \rightarrow 0$ fast enough, then $A\left(G_{1}\right)$ and $A\left(G_{2}\right)$ are isomorphic. For instance $\left\{\xi_{k}^{(i)}\right\}$ could be a sequence $\left\{\eta_{i}^{p(k)}\right\}$ of powers of a transcendental number $\eta_{i}$.

The arguments for Theorems 1 and 2 may be modified to deal with many sets not of the simple, convenient type described in $\S 5$. For example, we may allow each $E^{k}$ (and $F^{k}$ ) to be made up of intervals of various lengths, with $d_{k}^{\prime}$ (and $d_{k}$, respectively) as a bound rather than as the common value.

There exists a sec $E$ with the following properties: (1) except for the variation just mentioned, $E$ is of the type described in $\S 5$, with $J(k)=2^{k}$, such that (2) $E$ satisfies Condition II; (3) the points of $E$ are linearly independent over the rationals; and (4) $E$ is a set of multiplicity in the strict sense (and hence not a Helson set-cf. [7], Ch. XI, Theorem V). Rudin ([12]; cf. also [7], p. 103) constructed a set with properties (3) and (4), and (1) and (2) are easily assured in his procedure. Let $F$ be contructed as in $\S 5$, such that Condition I is satisfied, $J(k)=2^{k}$, and the sequence $\left\{s_{1}, s_{2}, \cdots\right\}$ is independent over the rationals. Then since $B\left(s_{1}, \cdots, s_{J(k)}\right)=1$ for every $k, F$ is a Helson set by Theorem 2, Corollary 4 (and hence a set of uniqueness in the broad sense-(cf. [7], Ch. XI, Theorem V). Let $E$ be the set of Rudin just described, and define $\varphi: F \rightarrow E$ in the manner of $\S 5$, taking $\varphi\left(s_{j}\right)=r_{j}$. Since both $\left\{s_{j}\right\}$ and $\left\{r_{j}\right\}$ are independent, Condition III is satisfied and by Theorem $1, \varphi$ is an isomorphism of $A(E)$ into $A(F) \cong C(F)$. The map cannot be surjective, for then $E$ would be a 
Helson set. The map $\Phi^{*}$ maps $N(F) \cong M(F)$ continuously into $N(E)$, and onto $M(E)$. It is notable that $\Phi^{*}$ thus must map some measures which are not pseudofunctions into the nonempty class $M(E) \cap P F$.

10. Some questions. We say that $\varphi \in A(F)$ is trivial if near each point of $F, \varphi(x)=r x+x_{0}$ for some real $r$ and $x_{0}$. No example is known of a nontrivial $\varphi \in A(F)$, taking $F$ into the circle, with $\sup _{n}\left\|e^{i n \varphi}\right\|_{\boldsymbol{A}(F)}<\infty$, where $F$ is a set of multiplicity.

Consider the sets

$$
E\left\{t_{j}\right\}=\left\{\sum_{j=1}^{\infty} x_{j} t_{j}: x_{j}=0 \text { or } 1\right\},
$$

where $t_{j} \rightarrow 0$ as $j \rightarrow \infty$. Perhaps it is the case that whenever $t_{j} \rightarrow 0$ and $t_{j}^{\prime} \rightarrow 0$ fast enough (in some sense that disregards arithmetic properties of the sequences), then the sets $E\left\{t_{j}\right\}$ and $E\left\{t_{j}^{\prime}\right\}$ have isomorphic restriction algebras.

Consider the compact group $X$ which is the complete direct sum of a countably infinite number of copies of the group $\{0,1\}$ under addition modulo 2. The elements of $X$ are the sequences

$$
\left\{\left(x_{1}, x_{2}, \cdots\right): x_{j}=0 \text { or } 1\right\} \text {. }
$$

Let $Y$ be the dual group of $X$, and let $A(X)$ be the Gel'fand representation of $L^{1}(Y)$. When, if ever, is the restriction algebra of a set (10.1) isomorphic to $A(X)$ ?

Added in proof: H. P. Rosenthal (cf. §1, Projections onto translation-invariant subspaces of $L^{p}(G)$, Memoirs of the A. M. S. No. 63, 1966) has shown that such an isomorphism never occurs.

Consider the quantity

$$
\||f|\|=\sup \left\{\frac{|(f, \mu)|}{\|\mu\|_{P M}}: \mu \in M(E), \mu \neq 0\right\} .
$$

If $f \in A(E)$, of course, \|\|$f\|\|\|f\|_{A^{(}(E)}$. How can we characterize the sets $E$ which have the property that

$$
\|\| f \|<\infty \Rightarrow f \in A(E)
$$

whenever $f \in C(E)$ ? Only recently, Katznelson constructed a set for which this implication fails. We shall here establish a sufficient condition for (10.2) to hold. The ideas are essentially those of the de Leeuw and Katznelson [4] and Kreìn ([1], §77); Krein proved (10.2) in the case when $E$ is an interval. Let $f \in C(E)$ and suppose $\|\mid f\|$ is finite. Then $f$ provides a bounded linear functional on $M(E)$ taken as 
a subspace of $P M$. Let $g \in P M^{*}$ be an extension of $f$ with norm $\|g\|=\|\| f \|$. Then $g$ may be decomposed, $g=g_{1}+g_{2}$ where $g_{1} \in A$, $g_{2} \in P F^{\perp}$, and $\|\mid f\|=\|g\|=\left\|g_{1}\right\|+\left\|g_{2}\right\|$. Since $g_{2} \in P F^{\perp}$, it has the property that

$$
\left|\left(g_{2}, S\right)\right| \leqq\left\|g_{2}\right\| \cdot \lim \sup _{|n| \rightarrow \infty}|\widehat{S}(n)| \text { for all } S \in P M .
$$

Since clearly $\left\|\left|g_{i}\|\mid \leqq\| g_{i} \|\right.\right.$ for $i=1$ and 2 , and $\left.\left.\||f|\|=\left\|g_{1}\right\|\right|+\right\| g_{2} \|$, it follows that ||$\left|g_{2}\right|||=\left|\left\|g_{2}\right\|\right|=\left|\| f-g_{1}\right| \mid$. To establish the implication (10.2), it suffices to show that always $g_{2}=0$. The situation is as follows:

$$
\begin{gathered}
\left(f-g_{1}-g_{2}, \mu\right)=0 \text { for } \mu \in M(E) ; \\
\left|\left\|f-g_{1} \mid\right\|=\left\|g_{2}\right\| ;\right. \\
\left|\left(f-g_{1}, \mu\right)\right| \leqq|| f-g_{1}||\left|\cdot \lim \sup _{|n| \rightarrow \infty}\right| \widehat{\mu}(n) \mid \text { for all } \mu \in M(E) .
\end{gathered}
$$

It follows that if every portion of the set $E$ is a set of multiplicity in the strict sense, and thus supports a nonzero, positive measure $\mu \in P F$, then (10.2) holds. For if $f-g_{1} \neq 0$, then $\left(f-g_{1}, \mu\right)$ would have to be nonzero for some $\mu \in M(E) \cap P F$-impossible, by (10.3). More generally, if for some $\eta>0, M(E)$ contains enough measures $\mu$ with

$$
\|\mu\|_{P M}=1 \text { and } \lim \sup _{|n| \rightarrow \infty}|\widehat{\mu}(n)| \leqq 1-\gamma
$$

to insure that $|\|f\|| \mid$ equals the supremum of $|(f, \mu)|$ over such $\mu$, then (10.3) gives a contradiction unless $g_{2}=0$, so that (10.2) must hold. It can be shown that this more general hypothesis is satisfied by the Cantor set.

The author wishes to thank his adviser, Yitzhak Katznelson, for his counsel and encouragement; and to acknowledge valuable conversations with Jean-Pierre Kahane, I. I. Hirshman, Jr., William G. Badé, and Robert Schneider. The author is also grateful to a prompt and helpful referee.

\section{REFERENCES}

1. N. Akhiezer, Theory of Approximations, translated by C. J. Hyman, Ungar, New York, 1956.

2. A. Beurling and H. Helson, Fourier-Stieltjes transforms with bounded powers, Math. Scand. 1 (1953), 120-126.

3. J. W. S. Cassels, An Introduction to Diophantine Approximation, Cambridge University press, 1957.

4. K. DeLeeuw and Y. Katznelson, On certain homomorphisms of quotients of group algebras, Israel Journal of Mathematics 2 (1964), 120-126. 
5. J. Dixmier, Sur un théorème de Banach, Duke Math. J. 15 (1948), 1057-1071.

6. N. Dunford and J. T. Schwartz, Linear Operators, Part I: General Theory, Interscience Publishers, New York, 1958.

7. J.-P. Kahane and R. Salem, Ensembles Parfaits et Séries Trigonométriques, Hermann, Paris, 1963.

8. L. Loomis, An Introduction to Abstract Harmonic Analysis, D. Van Nostrand, Princeton, 1953.

9. O. C. McGehee, An account of a result of Pyateckǐ̌-Šapiro (mimeographed), Dept. of Math., University of California at Berkeley, 1966.

10. I. I. Pyateckiì-Šapiro, Supplement to the work, "On the problem of uniqueness of expansion of a function in a trigonometric series" (in Russian), Učenye Zapiski Mosk. 165, Mat. 7 (1954), 78-97.

11. W. Rudin, Fourier Analysis on Groups, Interscience Publishers, New York. 1962. 12. - Fourier-Stietjes transforms of measures on independent sets, Bull. Am. Math. Soc. 66 (1960), 199-202.

13. A. Zygmund, Trigonometric Series, I and II, Cambridge University Press, 1959.

Received May 19, 1966. This work was supported mainly by the U. S. National Science Foundation Graduate Fellowship Program while the author was a student at Yale, and also by N. S. F. GP-4020 and GP-5585. 


\section{PACIFIC JOURNAL OF MATHEMATICS}

\section{EDITORS}

H. SAMELSON

Stanford University

Stanford, California

J. P. JANS

University of Washington

Seattle, Washington 98105
J. DugundJI

University of Southern California

Los Angeles, California 90007

RICHARD ARENS

University of California

Los Angeles, California 90024

\section{ASSOCIATE EDITORS}

E. F. BECKENBACH

B. H. NeumanN

F WolF

K. YOSIDA

\section{SUPPORTING INSTITUTIONS}

UNIVERSITY OF BRITISH COLUMBIA

CALIFORNIA INSTITUTE OF TECHNOLOGY

UNIVERSITY OF CALIFORNIA

MONTANA STATE UNIVERSITY

UNIVERSITY OF NEVADA

NEW MEXICO STATE UNIVERSITY

OREGON STATE UNIVERSITY

UNIVERSITY OF OREGON

OSAKA UNIVERSITY

UNIVERSITY OF SOUTHERN CALIFORNIA
STANFORD UNIVERSITY

UNIVERSITY OF TOKYO

UNIVERSITY OF UTAH

WASHINGTON STATE UNIVERSITY

UNIVERSITY OF WASHINGTON

AMERICAN MATHEMATICAL SOCIETY CHEVRON RESEARCH CORPORATION TRW SYSTEMS

NAVAL ORDNANCE TEST STATION

Printed in Japan by International Academic Printing Co., Ltd., Tokyo Japan 


\section{Pacific Journal of Mathematics}

\section{Vol. 21, No. $1 \quad$ November, 1967}

Friedrich-Wilhelm Bauer, Der Hurewicz-Satz................... 1

D. W. Dubois, A note on David Harrison's theory of preprimes . ......... 15

Bert E. Fristedt, Sample function behavior of increasing processes with stationary, independent increments ..................... 21

Minoru Hasegawa, On the convergence of resolvents of operators....... 35

Søren Glud Johansen, The descriptive approach to the derivative of a set function with respect to a $\sigma$-lattice ....................... 49

John Frank Charles Kingman, Completely random measures ............ 59

Tilla Weinstein, Surfaces harmonically immersed in $E^{3} \ldots \ldots \ldots \ldots \ldots . . \ldots 9$

Hikosaburo Komatsu, Fractional powers of operators. II. Interpolation spaces ......................................... 89

Edward Milton Landesman, Hilbert-space methods in elliptic partial differential equations ...................................... 113

O. Carruth McGehee, Certain isomorphisms between quotients of a group algebra ........................................ 133

DeWayne Stanley Nymann, Dedekind groups .................. 153

Sidney Charles Port, Hitting times for transient stable processes ......... 161

Ralph Tyrrell Rockafellar, Duality and stability in extremum problems involving convex functions . ............................ 167

Philip C. Tonne, Power-series and Hausdorff matrices . . .............. 189 